\title{
Pulmonary Artery-Pulmonary Vein Fistula: Contrast Echocardiography using Agitated Saline for Indirect Evaluation of Adequacy of Surgical Repair
}

\author{
${ }^{1}$ Jitin Narula, ${ }^{2}$ Minati Choudhury, ${ }^{3}$ Ujjwal Kumar Chowdhary, ${ }^{4}$ Usha Kiran
}

\begin{abstract}
Direct right pulmonary artery-left atrial communications are rare causes of congenital cyanotic heart disease. Bypassing of the pulmonary circulation and the filtering function of the lung predisposes these patients to high risk of stroke or cerebral embolism. Contrast echocardiography using transesophageal echocardiography helps in detection and/or confirmation of diagnosis of these extracardiac shunts as two-dimensional echocardiography of the heart is essentially normal in such patients. Additionally, it can help in the assessment of adequacy of surgical repair in the postoperative period.
\end{abstract}

Keywords: Pulmonary artery-pulmonary vein fistula, Contrast echocardiography, Nontranspulmonary contrast agents, Bubble contrast.

How to cite this article: Narula J, Choudhury M, Chowdhary UK, Kiran U. Pulmonary Artery-Pulmonary Vein Fistula: Contrast Echocardiography using Agitated Saline for Indirect Evaluation of Adequacy of Surgical Repair. J Perioper Echocardiogr 2014;2(2):61-64.

\section{Source of support: Nil}

Conflict of interest: None declared

\section{INTRODUCTION}

Direct right pulmonary artery-left atrial communications were first described by Friedlich et al in $1950^{1}$ and are among very rare causes of right-to-left shunt. These are characterized by direct connections between branches of a pulmonary artery and left atrium resulting in central cyanosis, exertional dyspnea and if the shunt is large may even result in polycythemia. In addition, these patients are at high risk for stroke or cerebral abscess because these communications bypass the pulmonary capillary

\footnotetext{
${ }^{1}$ Senior Resident, ${ }^{2}$ Additional Professor, ${ }^{3}$ Professor

${ }^{4}$ Professor and Head

1,2,4 Department of Cardiac Anesthesiology, All India Institute of Medical Sciences, New Delhi, India

${ }^{3}$ Department of Cardiothoracic and Vascular Surgery, All India Institute of Medical Sciences, New Delhi, India

Corresponding Author: Jitin Narula, Senior Resident Department of Cardiac Anesthesiology, All India Institute of Medical Sciences, New Delhi, India, Phone: 011-27432326 e-mail: jatin.narula.13@gmail.com
}

bed, thus, the filtering function of the lungs is compromised, allowing emboli and bacteria to pass directly into the systemic circulation. Detection and assessment of these extracardiac shunts is thus essential for prevention of adverse neurological outcome.

Contrast echocardiography was first reported by Gramiak et $\mathrm{al}^{2}$ in 1968 and is based upon contrast effect of the microbubbles due to the difference in density at interface between gas contained microbubbles and surrounding tissue. It has now become an indespensible tool for cardiovascular imaging, as nontranspulmonary contrast agents containing micro air bubbles are unable to pass the pulmonary circulation and in patients with normal circulation no contrast agent would appear on the left side of the heart after a systemic venous injection of the contrast. We report the case of an extracardiac right to left shunt in the form of a congenital right pulmonary artery to pulmonary vein fistula which was successfully ligated surgically under cardiopulmonary bypass and contrast echocardiography using agitated saline confirmed the adequacy of surgical repair.

\section{CASE REPORT}

A 16-year-old boy weighing $30 \mathrm{~kg}$ was admitted to our hospital with a long standing history of progressive increase in bluish discoloration, shortness of breath grade III and palpitation. Physical examination confirmed central and peripheral cyanosis, clubbing of fingers, normally split second heart sound and a silent precordium with a grade II/VI systolic murmur. Baseline arterial oxygen saturation on room air using pulse oximetry was $62 \%$ and his hemoglobin concentration was $20.7 \mathrm{gm} / \mathrm{l}$.

The chest radiograph and electrocardiography (ECG) of the patient were normal. Transthoracic echocardiography (TTE) showed a fistula between the right pulmonary artery to the right pulmonary vein, with mildly dilated right atrium and right ventricle with normally sized left-sided heart chambers. Ejection fraction of the patient was 60 to $65 \%$ and all the heart valves were normal. Computed tomography (CT) angiography was done which showed a dilated left atrium (LA) and left ventricle (LV) with a thickened anterolateral papillary 
muscle of the LV. A fistula was noted between the right pulmonary artery (RPA) and the right lower pulmonary vein (RLPV) with evidence of aneurysmal dilatation at the fistula site. All pulmonary veins on the left-side drained into the LA via a common trunk. Multiple significant aortopulmonary collaterals (APCs) from the descending thoracic aorta (DTA) were also seen.

Institutional protocol for anesthesia management was followed. In the operating room, routine monitoring was established and, after induction of anesthesia, transesophageal echocardiography (TEE) (Philips iE33 Model, Bothell, WA, USA) probe was inserted.

\section{Intraoperative TEE}

Upper esophageal aortic valve short-axis view showed a dilated main pulmonary artery (MPA) and right pulmonary artery (RPA) measuring 22.7 and $17.2 \mathrm{~mm}$ respectively (Fig. 1, Video 1). The probe was then slightly pulled upward and traced along the course of the RPA turning

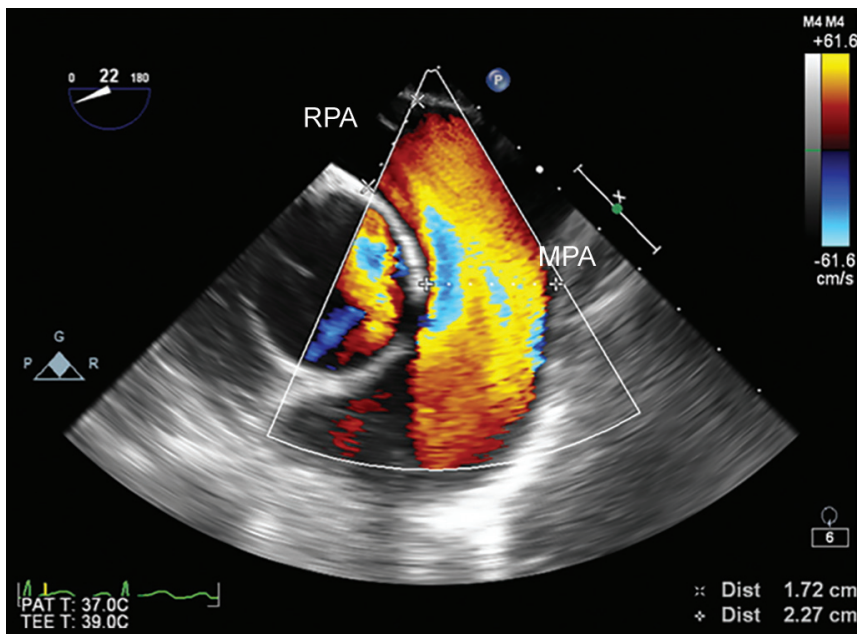

Fig. 1: Upper-esophageal aortic valve short-axis view showing the dilated main pulmonary artery and right pulmonary artery

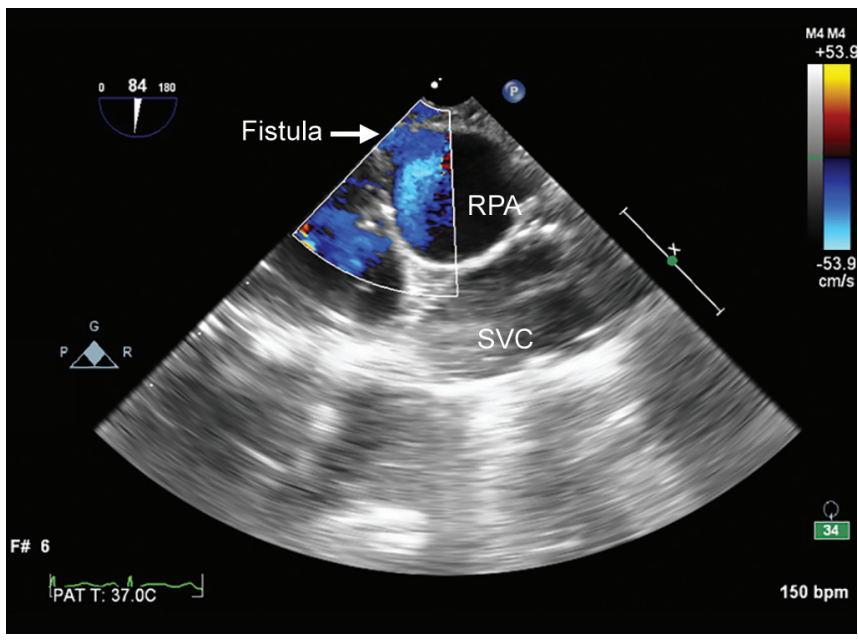

Fig. 3: Color flow Doppler showing the fistulous tract arising from the right pulmonary artery it toward the right which showed the ascending aorta, superior vena cava, right pulmonary artery and the left atrium (Fig. 2, Video 2). Color flow Doppler was applied which showed the fistulous tract to be arising from the RPA (Fig. 3, Video 3). TEE probe was further withdrawn slightly and rotated rightward which showed the aneurysmal dilatation at the fistula site and its communication with the RPA (Fig. 4, Video 4).

Contrast agent for bubble contrast echocardiography was produced by agitating solutions manually to form air-filled microbubbles using two $10 \mathrm{ml}$ syringes joined by a three-way stopcock. One syringe contained $10 \mathrm{ml}$ of $0.9 \%$ saline solution, and the other syringe contained $1 \mathrm{ml}$ of air. The saline solution was rapidly and repeatedly flushed back and forth between the two syringes through the three-way stopcock to agitate the solution. A mixture of air and liquid was thereby generated and injected through a central venous catheter placed in the right internal jugular vein.

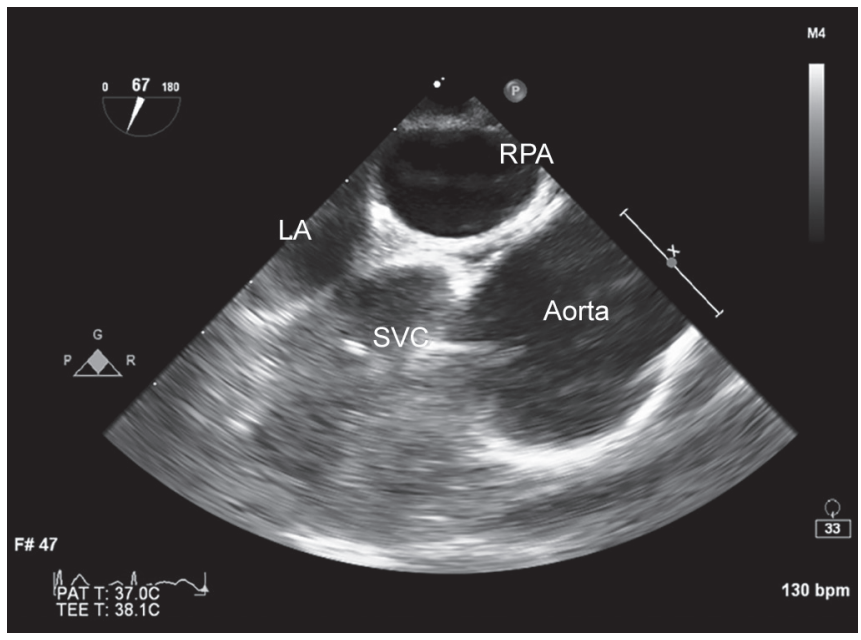

Fig. 2: Upper esophageal view with the probe pulled upwards and then traced along the course of the RPA showing the ascending aorta, superior vena cava, right pulmonary artery and left atrium

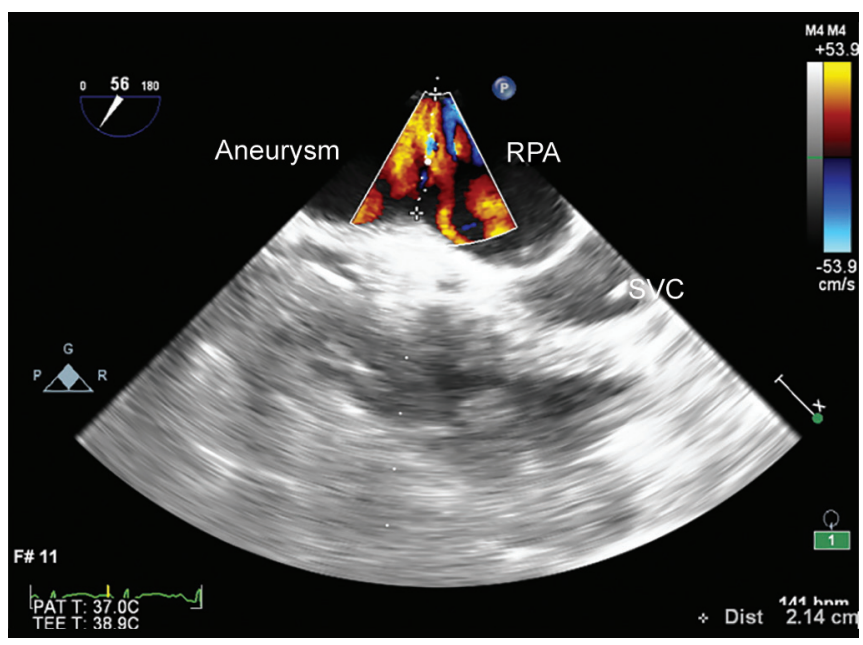

Fig. 4: Color flow Doppler showing the dilated fistulous tract arising from the right pulmonary artery 


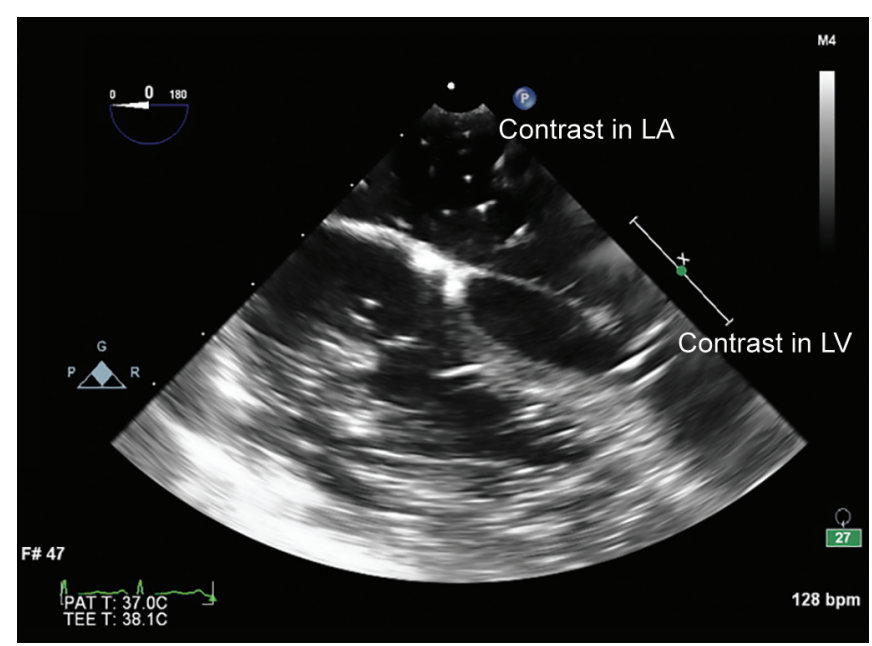

Fig. 5: Bubble contrast echocardiography using agitated saline, midesophageal four chamber view showing entry of contrast in the left atrium and left ventricle after injection into right atrium confirming the presence of an extracardiac shunt

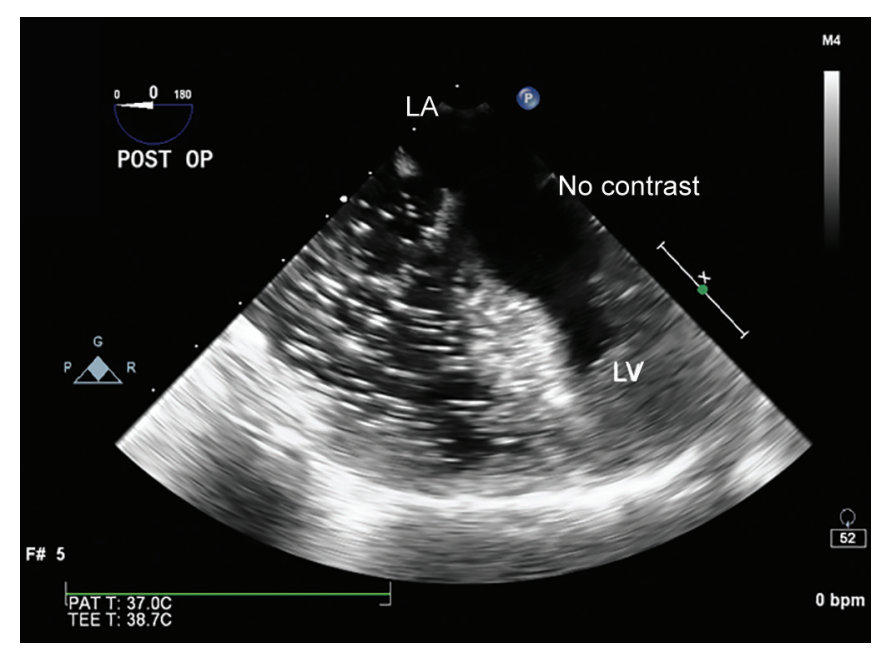

Fig. 7: Postoperative bubble contrast echocardiography with agitated saline failed to show any contrast in the left atrium and the left ventricle

The midesophageal four chamber view showed bubble contrast in the LA and LV and in the absence of any other identifiable septal defect, indicated a lack of passage of agitated saline solution through the pulmonary capillary bed and, therefore, was diagnostic of an extracardiac right-to-left shunt (Fig. 5, Video 5). Modified left pulmonary vein view showed the left-sided pulmonary veins entering normally into the left atrium. (Video 6).

Midline sternotomy and cardiopulmonary bypass (CPB) were used to aid the exposure of the fistula. The inferiorly placed fistulous tract between the RPA and the pulmonary vein (PV) (Fig. 6) was surgically ligated and the patient was weaned off $\mathrm{CPB}$ with a bypass time of 27 minutes. The arterial oxygen saturation in the pulse oximetry and $\mathrm{PaO}_{2}$ in the arterial blood after weaning off $\mathrm{CPB}$ on an inspired oxygen concentration of $50 \%$ were $100 \%$ and $178 \mathrm{~mm} \mathrm{Hg}$ respectively. Postoperative

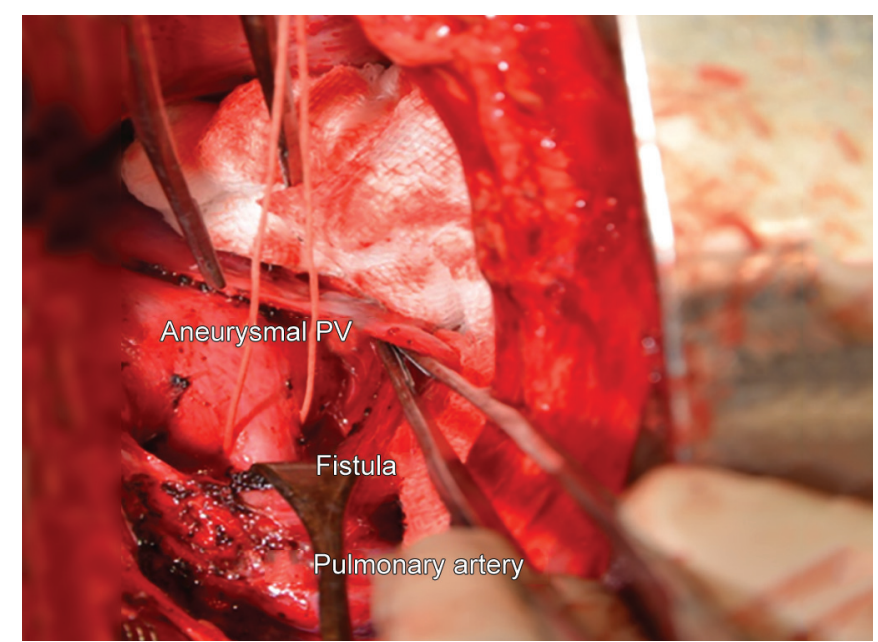

Fig. 6: Fistulous tract (looped) between right pulmonary artery and aneurysmally dilated right pulmonary vein

TEE was performed and as the fistulous tract could no longer be visualized, contrast echocardiography with agitated saline was repeated in the same manner which failed to show any contrast in the left atrium and the left ventricle indirectly ruling out presence of any residual mixing lesion (Fig. 7, Video 7).

After the completion of the surgery, the child was shifted to the intensive care unit (ICU) as per the institutional protocol and was extubated after 3 hours of elective mechanical ventilation. The child was discharged from ICU on postoperative day 2 and subsequently discharged home after 5 days following an uneventful postoperative course.

\section{DISCUSSION}

Pulmonary artery to left atrial communications are rare right to left type of shunts characterized by a direct communication between the pulmonary artery trunk or its branches and the left atrium. Patients usually present with central cyanosis, clubbing and cardiomegaly in childhood or adolesence. Neurological complications may include migraine $43 \%$, transient ischemic attack $37 \%$, stroke $18 \%$, abscess $9 \%$ and seizure $8 \%$. In our case, the child presented with dyspnea, dizziness, a gradually increasing cyanosis and clubbing of fingers.

Ohara et $\mathrm{al}^{3}$ classified these into four types based upon the presence or absence of an aneurysm in the fistula and upon the anatomy of the pulmonary venous drainage.

- Type 1: Normal pulmonary venous connection.

- Type 2: Lower lobe branch of the RPA communicates with an aneurysmally dilated right inferior PV.

- Type 3: All pulmonary veins drain into aneurysmal patch between the RPA and the LA.

- Type 4: A very proximal pulmonary arteriovenous malformation joins the LA. The right pulmonary vein 
joins the fistulous tract and the left PV drain directly into the LA.

Pulmonary angiography is considered as gold standard and provides best delineation of anatomical details as it is difficult to visualize the RPA to LA fistula on preoperative TTE. Bubble contrast echocardiography however compared with the traditional method of pulmonary angiography has shown to be a more sensitive tool in detecting early development of pulmonary arteriovenous malformations in patients with cavopulmonary anastomosis. ${ }^{4}$ Appearance of microbubbles in the left atrium after 2 to 5 seconds from opacification of the right atrium is suggestive of PAVMs. ${ }^{5}$ Since contrast microbubbles with a diameter $\geq 9 \mu \mathrm{m}$ do not pass the pulmonary capillary circulation, any appearance of intravenously injected microbubbles in the left side of the heart is considered positive for a right to left shunt. Similar left-sided echocardiographic appearance of microbubbles upon internal jugular venous injection in our patient helped us to confirm the diagnosis of an extracardiac right to left shunt. Additional provocative maneuvers, such as valsalva or coughing or abdominal compression may also be used to disclose transient right to left shunts.

Treatment options for our patient included angiography-guided transcatheter device closure or direct surgical closure. ${ }^{6,7}$ However, due to an aneurysmally dilated thin-walled fistulous communication and for the risk of pulmonary venous drainage obstruction by a large device, device closure was considered inappropriate and patient was taken up for direct surgical closure. CPB was initiated using aortobicaval cannulation and this facilitated a better surgical plane for excision and looping of the fistulous tract. Surgical ligation was carried out on beating heart with real-time guidance of TEE. In the postoperative TEE, the fistulous communication could no longer be visualized. The adequacy of the surgical repair was thus confirmed by the contrast echocardiography using nontranspulmonary contrast which showed absence of any contrast in left-sided heart chambers after injection into the central venous catheter which thus ruling out the presence of any residual extra cardiac shunt.

\section{CONCLUSION}

Transesophageal echocardiography using bubble contrast echocardiography is accurate for detection, confirmation of diagnosis and postoperative assessment of adequacy of surgical repair of cardiovascular shunts, especially in cases of extracardiac shunts and is thus a safe, simple, noninvasive, feasible and reproducible imaging technique.

\section{REFERENCES}

1. Friedlich A, Bing RJ, Blount SG Jr. Physiological studies in congenital heart disease, circulatory dynamics in the anomalies of venous return to the heart including pulmonary arteriovenous fistula. Bull Johns Hopkins Hosp 1950;86(1):20-57.

2. Gramiak R, Shah PM, Kramer DH. Ultrasound cardiography: contrast studies in anatomy and function. Radiology 1969; 92(5):939-948.

3. Ohara H, Ito K, Kohguchi N, et al. Direct communication between the right pulmonary artery and the left atrium: a case report and review of the literature. J Thorac Cardiovasc Surg 1979;77(5):742-747.

4. Chang RK, Alejos JC, Atkinson D, Jensen R, Drant S, Galindo A, Laks $\mathrm{H}$. Bubble contrast echocardiography in detecting pulmonary arteriovenous shunting in children with univentricular heart after cavopulmonary anastomosis. J Am Coll Cardiol 1999;33(7):2052-2058.

5. Gossage JR. The role of echocardiography in screening for pulmonary arteriovenous malformations. Chest 2003;123(2): 320-322.

6. Chowdhury UK, Kothari SS, Airan B, Subramaniam KG, Venugopal P. Right pulmonary artery to left atrium communication. Ann Thorac Surg 2005; 80(1):365-370.

7. Uthaman B, Al-Qbandi M, Abushaban L, Rathinasamy J. Transcatheter closure of large pulmonary arteriovenous fistula including pulmonary artery to left atrial fistula with Amplatzer septal occluder. Catheter Cardiovasc Interv 2007; 70(3):422-428. 